\title{
A Clinical Study of Retinal Changes in Chronic Renal Failure
} Patients

\author{
Dr. B. Manjula M.S. ${ }^{1}$, Dr. Muzafer Hussain Khadari M.S ${ }^{2}$., Dr. Soujanya \\ Velampalli ${ }^{3}$, \\ ${ }^{1}$ Assistant Professor of Ophthalmology, Guntur Medical College, GGH, Guntur. \\ ${ }^{2}$ Assistant Professor of Ophthalmology, Guntur Medical College, GGH, Guntur. \\ ${ }^{3} J u n i o r$ Resident, Guntur Medical College, GGH, Guntur
}

\begin{abstract}
:
Introduction: Chronic renal failure (CRF) affects every organ including eye. The prevalence is increasing all over world. Retinal changes were blamed to be the main culprit responsible for deterioration of vision in CRF patients.

Aim: We aimed to prospectively analyse the retinal changes among CRF patients. It is intended to screen patients for any visual threat so that necessary advise can be given before they become visually impaired.

Subjects and Methods: The present study was done from October 2016 to March 2017 on 60 consecutive patients who were admitted in Nephrology ward G.M.C /GGH, Guntur. Patients were staged using Cockcrouft-Gault formula into stage 1-5.

Results : When the results are analysed 40-60 yrs age group(70\%) is commonly affected with a definite male preponderance(70\%).The most common etiological factor leading to CRF in these patients is diabetes mellitus(32\%) followed by combined form (diabetes and hypertension)(29\%),followed by hypertension(25\%) and some are due to glomerulonephritis and polycystic kidney disease. Most of the patients in the study are with stage 2 \& 3 CRF. Diabetic retinopathy(32\%) is the most common finding followed by combined manifestation(29\%),followed by hypertensive retinopathy(25\%).The visual impairment was more due to diabetic retinopathy than hypertensive retinopathy. In patients having both diabetes and hypertension often retinopathy was worse. The severity of retinopathy was more as the renal disease progressed.

Conclusion: Retinopathy is often asymptomatic in its most treatable stage. Delay in diagnosis can result in significant increase in patients risk of visual loss . Ocular screening of CRF patients in their early stages is important as significant number of patients in this study required and were advised treatment so that irreversible visual impairment could be prevented.
\end{abstract}

Keywords: retinopathy, chronic renal failure, diabetes mellitus, hypertension

\section{Introduction}

Chronic kidney disease (CKD) is a worldwide public health problem, both for the number and cost of patient treatment involved The prevalence is increasing all over the world . It is a progressive and irreversible process that results in reduction of nephron number leading to end stage renal disease where patient has to be dependent on renal replacement therapy for survival $(1,2)$. The global annual growth of number of End Stage Renal Disease (ESRD) patients is at $7 \%(3,4)$. It accounts for $60 \%$ of all deaths worldwide. Many studies described the high prevalence of retinal changes in patients with CRF which were blamed to be the main culprit responsible for deterioration of vision in CRF patients. Micro vascular disease may play a role in the development of ocular and renal disease. Therefore the presence of retinal pathology may provide information of prognostic value regarding progression of renal disease. Basement membrane thickening, a common pathogenic mechanism may explain the relationship to some extent(5).

Various causes for diminished visual acuity in these patients are due to worsening of diabetic and hypertensive retinopathy, ischaemic optic neuropathy, central retinal vein occlusion and cortical blindness Bright in 1836 was the first to associate renal disease with blindness(6). CRF is defined as Glomerular Filtration Rate (GFR) of less than $60 \mathrm{ml} / \mathrm{mt} / 1.73 \mathrm{~m} 2$ lasting for longer than 3 months or kidney damage(7) Glomerular filtration rate (GFR) calculation: Ux V/P where $\mathrm{U}=24$ hour urinary creatinine, $\mathrm{V}=24$ hour urinary volume and $\mathrm{P}=$ serum creatinine. Based on guide lines of National Kidney Foundation, stages of CKD are defined according to the estimated GFR.

\section{Staging Of CRF done Using Cockcrouft-Gault Formula which Is: \\ Estimated creatinine clearance $(\mathrm{ml} / \mathrm{mt})=(140$-age $) \times$ body weight $(\mathrm{kg}) / 72 \times \mathrm{Pcr}(\mathrm{mg} / \mathrm{dl})$ Stage $0(>90 \mathrm{GFR}$ $\mathrm{ml} / \mathrm{min} / 1.73 \mathrm{~m} 2)$, Stage $1(90 \mathrm{GFRm} / \mathrm{min} / 1.73 \mathrm{~m} 2)$ Stage 2(60-89 GFR ml/min/1.73m2), Stage 3(30-59GFR}


$\mathrm{ml} / \mathrm{min} / 1.73 \mathrm{~m} 2)$, Stage $4(15-29 \mathrm{GFR} \mathrm{ml} / \mathrm{min} / 1.73 \mathrm{~m} 2)$ and Stage $5(<15 \mathrm{GFR} \mathrm{ml} / \mathrm{min} / 1.73 \mathrm{~m} 2)$

The most common cause of CRF is diabetes mellitus followed by hypertensive nephroangiosclerosis, and various primary and secondary glomerulo nephropathies. Ocular morbidity may be directly due to hypertension, uremia and anaemia. Retinopathy is often asymptomatic in its most treatable stage . Delay in diagnosis can result in significant increase in patients risk of visual $\operatorname{loss}(8)$. Ocular condition is also an indicator of the metabolic control of the disease process.

\section{Subjects And Methods}

This is a prospective, cross sectional descriptive hospital based study, where data was collected from 60 patients with CRF during a period of six months from October 2016 to March 2017 who were admitted to the nephrology ward at Government General Hospital, Guntur. The study included CRF disease patients attended to nephrology speciality. Cases of reversible renal failure and patients on dialysis were excluded. A prepared proforma was used to collect data which includes history taking, BCVA by Snellens chart, detailed slit lamp examination of anterior segment, IOP measurement, posterior segment examination using $+90 \mathrm{D}$ lens and fundus photography. Hypertensive retinopathy was graded based on Keith-Wegener classification, Diabetic retinopathy and macular edema were classified on the basis of ETDRS(early treatment diabetic retinopathy study). Other investigations done for this study are $\mathrm{Hb} \%$, TC , DC, ESR, blood urea serum creatinine, serum calcium ,serum phosphorous, serum sodium, potassium, lipid profile, FBS,PPBS, urine examination.

\section{Results}

Among total admissions (from Oct 2016-March 2017) of 543, CRF contributed to 65\%(353) in nephrology dept. 60 patients of CRF who were admitted to nephrology department and met the inclusion and exclusion criteria were included in the study. Highest no of patients were in the age group between 40-60 yrs, with mean age of 53.3 and a standard deviation of 4.9 years which is consistent with the study done by L.Bajracharya et al (48.3+-) 14.9years. Among 60 group, males constituted 42 and females constituted 18.The ratio is 2.3:1 which is consistent with the study of L.Bajracharya et al who observed the male: female ratio 2.3:1.

In the present study majority of patients with CRF had diabetes (32\%) followed by patients with diabetes and hypertension (29\%), followed by hypertension which constituted 25\%.These results are not in accordance with the study of L.Bajracharya et al where the commonest cause of CRF was HTN 43 out of 119(36.1\%), followed by DM (27.7\%) and glomerulonephritis(20.2\%).In the present study stage 1,2 CRF accounted for 19\%, stage $3 \mathrm{CRF}$ accounted for $33 \%$,stage $4 \mathrm{CRF}$ for $36 \%$ and stage 5 for $12 \%$. Assessment of visual acuity in eyes taken individually showed that $23 \%$ of the 120 eyes had visual acuity ranged between $6 / 6-6 / 18,49.5 \%$ had between $6 / 24-6 / 69$ and $27.5 \%$ had less than $6 / 60$. Out of 92 eyes with visual acuity $<6 / 18$ the most common cause was maculopathy $(41.55 \%)$ followed by cataract $(40.25 \%)$. These values are higher to the observation seen by L.Bajracharya et al which showed maculopathy as the cause of visual impairement in 23 eyes out of 238 eyes $(9.7 \%)$, followed by cataract in $14(5.9 \%)$ eyes, PDR in $9(3.8 \%)$ eyes. Prevalence of retinal pathology was highest among patients with diabetes (32\%), followed by both diabetes and hypertension $(29 \%)$ followed by patients with hypertension (25\%). There was one case of severe CRF with bilateral exudative type of retinal detachment. It is stated that in uremia, retinal detachment should be regarded as an immediate indication for peritoneal and hemodialysis (9) While comparing grades of hypertensive retinopathy with CRF, majority of patients had Grade II hypertensive retinopathy (26\%) followed by Grade III hypertensive retinopathy(25\%). Grade 4 hypertensive retinopathy was present in one patient with stage $5 \mathrm{CRF}$. In this study majority of patients had moderate, severe, very severe NPDR that accounted for 13\%,14\%,15\% respectively. Patients with PDR and high risk PDR had stage 3,4 and 5 CRF.

Table 1: Distribution of patients according to their age group

\begin{tabular}{|c|c|c|c|}
\hline Age Group & No. of Cases & Mean Age & $\begin{array}{c}\text { Standard } \\
\text { Deviation }\end{array}$ \\
\hline $0-20$ & $2(3 \%)$ & 15.0 & 5.0 \\
\hline $21-40$ & $9(15 \%)$ & 32.2 & 6.6814669 \\
\hline $41-60$ & $42(70 \%)$ & 53.3043478 & 4.91222964 \\
\hline $61-80$ & $7(11.6 \%)$ & 69.2857143 & 4.50921279 \\
\hline
\end{tabular}

Table 2: BCVA

\begin{tabular}{|l|c|c|c|c|c|c|}
\hline BCVA & $\begin{array}{c}\text { Stage } 1 \& 2 \\
23 \text { eyes }\end{array}$ & Stage 3 & Stage 4 & Stage 5 & Total & $\%$ \\
\hline$\geq 6 / 18$ & 8 & 7 & 43 eyes & 14 eyes & 120 eyes & \\
\hline $6 / 24-6 / 60$ & 13 & 21 & 20 & 4 & 28 & $23 \%$ \\
\hline$<6 / 60$ & 2 & 12 & 14 & 5 & 59 & $49.5 \%$ \\
\hline
\end{tabular}


$P$ value $=0.117$ not significant

Table 3: Etiology of visual impairment

\begin{tabular}{|l|c|c|}
\hline \multicolumn{1}{|c|}{ Causes } & No. of Eyes & $\begin{array}{c}\text { Percentage of } \\
\text { Total Eyes }\end{array}$ \\
\hline Cataract & 37 & $40.25 \%$ \\
\hline Maculopathy (HTN, D.M) & 38 & $41.55 \%$ \\
\hline PDR & 6 & $5.80 \%$ \\
\hline Retinal Detachment & 1 & $1.29 \%$ \\
\hline ARMD & 1 & $1.29 \%$ \\
\hline Others & 9 & $9.69 \%$ \\
\hline TOTAL & 92 & \\
\hline
\end{tabular}

Table 4:

\begin{tabular}{|l|c|c|c|c|c|c|}
\hline \multicolumn{1}{|c|}{ Ocular Findings } & Stage 1\&2 & Stage 3 & Stage 4 & Stage 5 & Total & $\%$ \\
\hline $\begin{array}{l}\text { Diabetic } \\
\text { Retinopathy }\end{array}$ & 4 & 5 & 7 & 3 & 19 & $32 \%$ \\
\hline $\begin{array}{l}\text { Hypertensive } \\
\text { Retinopathy }\end{array}$ & 4 & 6 & 3 & 1 & 14 & $25 \%$ \\
\hline Mixed & 2 & 5 & 9 & 1 & 17 & $29 \%$ \\
\hline BRVO & 0 & 0 & 1 & 0 & 1 & $0.6 \%$ \\
\hline ARMD & 0 & 1 & 0 & 0 & 1 & $0.6 \%$ \\
\hline Disc Pallor & 0 & 0 & 0 & 1 & 1 & $0.6 \%$ \\
\hline Glaucoma Suspect & 0 & 1 & 0 & 0 & 1 & $0.6 \%$ \\
\hline Bullous RD & 0 & 0 & 0 & 1 & 1 & $0.6 \%$ \\
\hline
\end{tabular}

\section{Discussion}

CRF is a slowly progressive deterioration of renal function and it can occur at any age group. In the present study majority of patients are between the age group of 41-60 yrs with a mean age of 53.3 yrs and a standard deviation of 4.9 years which constituted $69 \%$ of total cases. Out of 60 patients with chronic kidney disease , 41 are males and 19 are females the ratio being 2.15:1 which was similar to world wide data(1).The reason may be due to faster rate of deterioration of kidney function with some forms of glomerulonephritis and polycystic kidney disease.(table1). A report from Atherosclerosis Risk in Communities (ARIC) study (10) demonstrated a strong association between retinopathy features and renal dysfunction that was independent of age , diabetes, hypertension, and other risk factors.

In the present study the most common etiological factor leading to CRF was diabetes mellitus followed by both diabetes and hypertention followed by hypertension alone that constituted 19,17,14 patients out of 60 patients respectively. These observations are not consistent with the study of L.Bajracharya et al where the commonest cause of CRF was hypertension 43 out of 119(36.1\%), followed by diabetes mellitus (27.7\%), and glomerulonephritis $(20.2 \%)(11)$ which may be due to geographical in the prevalence and etiology of CRF.

Diminished vision is the most common ocular symptom observed in 37 out of 60 which correlates with study by L. Bajracharya et al. In the present study out of 60 eyes examined BCVA >/ was present in $23 \%$ of eyes. $49.5 \%$ i.e 59 eyes had visual acuity between $6 / 24$ to 6/60. $27.5 \%$ i.e.33 eyes had BCVA less than 6/60. 77\% of 120 eyes examined therefore had visual acuity $<6 / 18$. This is higher than that of study by L. Bajracharya et al.(table 2,3$)$

The most common reason for the visual impairment was maculopathy (41.55\%) followed by cataract (40.25\%). This pathology is higher than in the general population. This supports the assumption that CRF increases the risk of developing maculopathy and cataract. This is not in agreement with the observations made by L. Bajracharya et al which showed maculopathy as cause of visual impairment in 23(9.7\%) eyes followed by cataract 14 eyes(5.9\%). The higher incidence of visual impairment due to diabetic retinopathy when compared to reports in literature is may be because of higher incidence and poor control of diabetes in our study patients .(table4)

Higher incidence of cataract may be explained by the presence of more number of patients in stage 3 and 4 in this study. Increased levels of oxidised glutathione in CRF patients in severe stages exposes the lens to oxidative stress which may be one possible reason. The higher incidence of diabetes among patients included in the study may be another possible reason for higher incidence of cataract.

\section{The most important and vision threatening findings were:}

a) Hypertensive retinopathy: In the present study $25 \%$ of total patients with CRF had hypertensive retinopathy. The findings in this study does not correlate with the studies done by L. Bajracharya et al (47.10\%), M Popa et al Single patient of stage 5 CKD had grade 4 hypertensive retinopathy with optic disc edema.

b) Diabetic retinopathy: In the present study $32 \%$ of total patients with CRF had diabetic retinopathy. Mild NPDR was seen mostly in stage 1 and $2 \mathrm{CRF}$ but moderate, severe and PDR were seen in higher stages of CRF All 
patients with diabetic nephropathy had diabetic retinopathy. Observations made in this study correlates with observations seen in other studies done by L. Bajracharya et al, Ley AM et al (12), T Schleiffer et al(13). The more severe forms of retinopathy are detected as renal disease progresses.

c) Combination form: In the present study $29 \%$ of patients with CRF had both diabetic and hypertensive retinopathy. Diabetic retinopathy was worse in patients who had both diabetes and hypertension.

d) Maculopathy: Out of 38 eyes with maculopathy, 36 were related to diabetic retinopathy and 2 eyes were associated with hypertensive retinopathy. Among 36 eyes with macular edema in diabetic retinopathy 32 eyes had clinically significant macular edema. Diabetic maculopathy was responsible for drop of vision more than hypertension because most of the diabetic retinopathies found in our patients were in advanced stages unlike hypertensive retinopathies which were present mostly in early stages.

e) Retinal detachment: There was one case of bilateral, exudative type of retinal detachment in patient with severe grade of renal disease.(14)

All the cases of grade 3 and grade 4 hypertensive retinopathy were detected for the first time during this study. Grade 3 and grade 4 hypertensive retinopathy has bad prognosis and this may alert the physician for more aggressive management of hypertension. Among the diabetic retinopathy patients there were 1 high risk PDR, 3 PDR, 8 cases of very severe diabetic retinopathy, 7 cases of severe diabetic retinopathy all never treated before and several patients were advised urgent Laser treatment for retina.

\section{Conclusion}

This study is an attempt to assess the retinal changes associated with CRF. The most common cause of CRF in our study is diabetes mellitus. Maculopathy is the most commonly observed cause for diminished vision. Hence every patient with CRF should undergo complete ophthalmic checkup and frequent follow up. Retinopathy is often asymptomatic in its early stages. Delay in diagnosis can result in significant increase in the patient's risk of vision loss. Ocular condition is an indicator of the metabolic control of the disease process. Measures like detailed ophthalmic examination, strict metabolic control of diabetes, and strict control of hypertension are important .

\section{References}

[1]. Weatherall DJ, Ledingham JGG, and Warrell DA. Oxford tex

[2]. book of medicine Vol III. 3rd ed. Oxford -New York-Tokyo:

[3]. Oxford Univ Press 1996; 3294-5.

[4]. Grossmann A, Gioberge S, Moeller S, et al. ESRD patients in 2004.global overview of patient numbers, treatment modalities and associated trends. Nephrol Dial Transplant . 2005;20(12):2587-93.

[5]. Reddy KS, Shah B, Varghese C, Ramadass A. Responding to the threat of chronic diseases in India. Lancet 2005;366:1744-1749.

[6]. Lysaght MJ. Maintenance dialysis population dynamics : current trends and long term implications. J Am Soc Nephrol 2002; 13: $37-40$

[7]. Olson J .: Diabetes mellitus. In : Hepinstall,s Pathology of Kidney, 5 th Ed., edited by Jennette JC, Olson JL, Schwartz MM, Silva FG ,editors. Philadelphia, Lippincott-Raven,1998, pp 1247-1287.

[8]. Duke-Elders S, Dohree JH. System of Ophthalmology, Vol

[9]. X .1st ed. London: The CV Mosby Company 1967;

[10]. chapter 4, 315-47.

[11]. Primer on kidney diseases, Arthur Greenberg, Alfred K.cheung, Thomas M. Coffman, Ronald J.Falk, J.Charles Jennette 5th Edition,Chapter 52,53

[12]. Albert DM, Jacobiec FA, Azar DT, Gragoudas ES,Power SM, Robinson NL. Principles and practice of Ophthalmology Vol II . 2nd ed.Philadelphia : WB Saunders 2000;1900-13.

[13]. Wong TY, Coresh J, Klein R, Muntner P, Couper DJ, Sharrett AR, Klein BE, HeissG, HubbardLD, Duncan B: Retinal microvascular abnormalities and renal dysfunction: The Atherosclerosis Risk in Communities Study. J Am Soc Nephrol 15:2469-76 2004.

[14]. L.Bajrachatya, DN Shah, KB Raut and S Koirala; Ocular evaluation in patients with CRF: Nepal Med Coll.j 2008 ;10(4);209-214.

[15]. Leys AM. Eye fundus of the diabetic patient with nephropathy and hypertensive retinopathy. Macroangiopathic complication. Bull Soc.Belge Ophtalmol.1995;256:49-59.

[16]. Schleiffer T, HolkenH, Brass H, Morbidity in 565 type 2 diabetic patients according to stage of nephropathy. J Diabetes complications 1998;12:103-9.

[17]. Liao HP, Yang KJ, Lai CC, Chen TL, Chen KS. Rapidly resorptive exudative retinal detachment in a patient with renogenic hypertension: case report. Changgeng Yi Xue Za Zhi 1999; 22: 324-7. 